\title{
Prevalência de obesidade, hipertensão e sedentarismo em alunos do Ensino Médio da rede pública da cidade de Gurupi
}

\author{
Prevalence of obesity, hypertension and sedentarism in Middle School students of the \\ Gurupi city public network
}

\author{
Jéssica Barreto Abreu Rodrigues", Daniele Gonçalves \\ Lisboa Gross²
}

\section{RESUMO}

Introdução: A obesidade presente na adolescência tem sido um motivo de alerta devido sua possível progressão para vida adulta e a atividade física nessa fase, tornase fator determinante na qualidade de vida do adulto e na incidência de hipertensão e sedentarismo. Objetivo: identificar a prevalência de obesidade, hipertensão e sedentarismo em alunos do ensino médio da rede pública da cidade de Gurupi. Material e Métodos: Trata-se de uma pesquisa de campo com característica transversal e descritiva, de natureza quantitativa. A amostra da pesquisa foi composta por 45 alunos do sexo masculino de 15 a 19 anos. Para levantamento dos dados analisou-se o índice de massa corporal (IMC); pressão arterial (PA); e o nível de atividade física por meio do questionário internacional de atividade física (IPAQ). Resultados: Os resultados foram analisados quanto à normalidade por meio do teste Shapiro Wilk; para a comparação dos dados descritivos por meio do teste Mann-Whitney; e análises comparativas através do teste de Poisson. Os resultados apresentaram uma prevalência 2,2\% de obesidade, $2,2 \%$ de hipertensão e $0 \%$ sedentarismo. Esses percentuais foram significativamente $(p<0,05)$ menores quando comprados aos valores encontrados em algumas pesquisas. Conclusão: Conclui-se que os valores encontrados em Gurupi são significativamente abaixo dos encontrados em referência a outras literaturas.

Palavras-chave: Obesidade. Hipertensão. Inatividade Física. Adolescentes.

\section{ABSTRACT}

Introduction: Obesity present in adolescence has been a reason for alertness due to its possible progression to adulthood and physical activity in this phase, becomes a determining factor in the quality of life of the adult and in the incidence of hypertension and sedentary lifestyle. Objective: identify the prevalence of obesity, hypertension and sedentarism in high school students of the public school in the city of Gurupi. Material and Methods: This is a field research with a cross-sectional and descriptive character, of a quantitative nature. The research sample consisted of 45 male students aged 15 to 19 enrolled. Data were analyzed for body mass index; blood pressure; and the level of physical activity through the International Physical Activity Questionnaire (IPAQ). The results were analyzed for normality using the Shapiro Wilk test. After comparing the descriptive; using the Mann-Whitney test; the other comparative analyzes were through the Poisson test. Results: The results showed a prevalence of $2.2 \%$ of obesity, $2.2 \%$ of hypertension and $0 \%$ of sedentary lifestyle. These percentages were significantly ( $p$ $<0.05$ ) lower when purchased from the values found in some studies. Conclusion: It is concluded that the values found in Gurupi are significantly below those found in reference to other literatures.
1Pós-Graduanda em Educação

Física aplicada ao Fitness e

Wellness, pela Universidade

de Gurupi - UnirG.

${ }^{2}$ Graduada em Educação

Física, Licenciatura plena pela

Universidade de Gurupi -

UnirG. Especialista em

Educação Física Escolar pelo

Instituto Específico de Ensino

Pesquisa e Pós-Graduação de

Gurupi-TO - IEP. Docente do

curso de Educação Física,

Licenciatura e Bacharelado,

da Universidade de Gurupi -

UnirG.

Keywords: Obesity. Hypertension. Physical inactivity. Adolescents. 


\section{INTRODUÇÂOO}

Sabe-se que a Revolução Industrial tornou a vida do homem mais fácil e o desenvolvimento da indústria e agricultura aumentou os avanços da tecnologia, e as maquinas começaram a fazer o que antes era feito à mão. Mas, com ausência de realizar atividades físicas diárias, o corpo tornou-se cada vez mais inativo e vulnerável, as doenças hipocinéticas advindas do sedentarismo e da obesidade, e estas que estão associadas à aquisição de doenças cardiovasculares como a hipertensão arterial.

Segundo Rique ${ }^{1}$ as doenças cardiovasculares tem sido uma das maiores causas de mortalidade no mundo. Sendo influenciados pelo estilo de vida das pessoas como nível de atividade física e hábitos alimentares, fatores esses que estão interligados a obesidade.

Devido à obesidade e a hipertensão serem atualmente um dos grandes problemas de saúde pública, países desenvolvidos têm juntados esforços na prevenção e tratamento destas doenças, onde têm sido criados planos para a redução do sedentarismo, através da pratica de atividade física regular, garantindo assim uma melhor saúde individual e coletiva. ${ }^{2}$

A obesidade presente na infância $e$ adolescência tem sido um motivo de alerta devido sua possível progressão para vida adulta. ${ }^{3}$ Assim estudos têm sido feitos comprovando que hábitos de atividade física na adolescência determinam parte dos níveis de atividade física na idade adulta. ${ }^{2}$

O Ministério da Saúde relata que segundo pesquisa feita pela Vigilância de Fatores de Risco e Proteção para Doenças Crônicas por Inquérito Telefônico. A hipertensão arterial alcança $23,3 \%$ da sociedade brasileira. Já a obesidade alcança $48,5 \%$ quase à metade população. Revela também que $25,8 \%$ das pessoas ficam mais de três horas assistindo televisão por dia. ${ }^{4}$

Esta pesquisa é relevante, pois pretende verificar a prevalência de fatores de risco de jovens, que associados com o sedentarismo e alimentação errada podem torna-se fatores preocupantes na transformação de possíveis adultos obesos, hipertensos entre outros danos à saúde. Para que possam ser criados programas para intervir, evitando o desenvolvimento de doenças como obesidade e a hipertensão, podendo dessa forma gerar adultos com um estilo de vida mais saudável.

Portanto, através dos fatos citados, o presente estudo teve como objetivo identificar a prevalência de obesidade, hipertensão e sedentarismo em alunos do ensino médio da rede pública da cidade de Gurupi e comparar os resultados com dados de referências de outros estudos. 


\section{MATERIAIS E METODOS}

Trata-se de uma pesquisa de campo com característica transversal e descritiva, e de natureza quantitativa. A pesquisa foi realizada em três Centros de Ensino Médio e duas escolas estaduais, sendo essas, instituições públicas da cidade de Gurupi - TO, as quais podem ser identificadas, como: Centro de Ensino Médio Ary Ribeiro Valadão Filho, Centro de Ensino Médio de Gurupi, Centro de Ensino Médio Bom Jesus, Escola Estadual Dr. Joaquim Pereira da Costa e Escola Estadual Setor Aeroporto. Os critérios de inclusão utilizados na realização do referido estudo, foram: ser aluno do sexo masculino, estar regularmente matriculado em uma das cinco unidades escolares citadas acima, ter idade $\geq$ 15 e $\leq$ a 19 anos, ser sorteado, aceitar participar do estudo e assinar o termo de consentimento livre e esclarecido, aos alunos menores de 18 anos, ter o termo de consentimento livre e esclarecido (TCLE) assinado pelos pais ou responsáveis. Foram excluídos do estudo os sujeitos que não responderam o questionário (IPAQ) adequadamente e não se enquadraram nos critérios de inclusão citados acima.

No universo de 2.848 de alunos de ambos os sexos matriculados nas cinco unidades escolares, foi realizada uma coleta preliminar com 8 sujeitos do sexo masculino com faixa etária de 15 a 19 anos. A partir desses dados coletados, foi identificado o erro e o desvio padrão. Em seguida, calculou-se o número de sujeitos necessários para que represente uma amostra significativa, através da fórmula citada por Barreto. ${ }^{5}$

Com a efetivação do cálculo descobriu-se a necessidade de coletar 31 sujeitos do sexo masculino, com a faixa etária entre 15 e 19 anos. Esse quantitativo foi dividido proporcionalmente pela quantidade de alunos matriculados em cada escola. Em seguida, a quantidade de alunos foi subdividida entre os períodos matutino, vespertino e noturno, também de forma proporcional. Para facilitar o processo de coleta de dados, e também para realizar uma coleta de forma mais heterogenia possível, foi adotado um sorteio, no qual selecionou um aluno de cada sala, de cada escola pública, que frequentasse o ensino médio. No total foram coletados 55 alunos do sexo masculino. No entanto, destes foram excluídos 10 alunos que não responderam o questionário (IPAQ) adequadamente, totalizando uma amostra de 45 voluntários.

Essa pesquisa seguiu todas as orientações estabelecidas na Resolução 466/2012 do Conselho Nacional de Saúde, foi realizada somente após aprovação do comitê de ética e pesquisa da Universidade de Gurupi - UnirG, e assinatura do voluntário ou responsável 
(se for de menor) do termo de consentimento livre e esclarecido (TCLE). O referido projeto de pesquisa foi submetido ao Comitê de Ética em Pesquisa da Universidade de Gurupi UnirG e foi aprovado sob o número 158/2011.

\section{RESULTADOS}

O município de Gurupi possui 5 unidades escolares de ensino médio regular de educação pública estadual e municipal, contabilizam 2.848 alunos de ambos os sexos, regularmente matriculados nas referidas unidades escolares. Participaram desta amostra 55 sujeitos do sexo masculino de 15 a 19 anos, no entanto, 10 sujeitos foram excluídos da pesquisa, devido apresentarem respostas equivocadas no questionário de atividade física, tal como, 540 minutos de exercício vigoroso por 5 vezes na semana, totalizando, enfim o quantitativo de 45 voluntários.

A apresentação dos resultados dar-se-á, nesse estudo, por meio de tabelas e gráficos. A tabela 1, a seguir, exibe características gerais da população estudada que subdivide-se em dois grupos de diferentes idades (Grupo 15-16 anos e Grupo 17-18 anos). Observou-se que os sujeitos, no geral, estão dentro dos padrões normais de índice de massa corporal (IMC), pressão arterial sistólica (PAS) e diastólica (PAD). Através da análise identificou-se que não houve diferença significativa entre os grupos de idade, no entanto, os valores tiveram uma tendência maior no grupo de 15-16 anos, com exceção da categoria da PAD, na qual, o valor teve uma tendência maior no grupo de 17-18 anos.

Tabela 1 - Media e desvio padrão da idade, índice de massa corporal e pressão arterial sistólica e diastólica de alunos do sexo masculino de unidade escolares de ensino médio público da cidade Gurupi.

\begin{tabular}{cccccc}
\hline & $\mathrm{N}(\%)$ & IDADE & IMC & PAS & PAD \\
\hline 15-18 anos & $45(100)$ & $16,6 \pm 0,96$ & $20,87 \pm 2,98$ & $114,89 \pm 8,15$ & $73,11 \pm 8,74$ \\
15-16 anos & $19(42,2)$ & $15,6 \pm 0,47$ & $21,07 \pm 3,63$ & $115,26 \pm 9,05$ & $71,58 \pm 9,58$ \\
17-18 anos & $26(57,8)$ & $17,3 \pm 0,49$ & $20,72 \pm 2,42$ & $114,62 \pm 7,61$ & $74,23 \pm 8,09$
\end{tabular}

$\mathrm{n}=$ número total de sujeito. $\mathrm{IMC}=$ índice de massa corporal. $\mathrm{PAS}=$ pressão arterial sistólica. $\mathrm{PAD}=$ pressão arterial diastólica. O teste de Mann-Whitney: amostras independentes foi utilizada para comparar as médias entre os grupos 15-16 anos e 17-18 anos.

Quanto aos resultados da pressão arterial, detectou-se que a prevalência da hipertensão, ilustrado como "Estágio I" no gráfico 1, foi de 2,2\% entre sujeitos pesquisados, quando subdivididos, nota-se que este valor ficou centrado apenas no grupo com idade de 
15-16 anos. O percentual encontrado neste estudo de 2,2\% é significativamente abaixo das expectativas de $7 \%$ sugeridas pelo III Consenso de Pressão Arterial da Sociedade Brasileira de Cardiologia.

No entanto, encontraram-se $48,9 \%$ de indivíduos entre 15-18 anos como "limítrofe" (pré-hipertensão), o mesmo percentual de sujeitos com a pressão arterial "normal". Sendo observado que a idade não foi um fator determinante, pois não houve diferença significativa entre os grupos de idade. Contudo notou-se uma tendência maior de limítrofes no grupo de 17-18 anos.

Gráfico 1 - Percentuais da classificação da pressão arterial de alunos do sexo masculino de unidade escolares de ensino médio público da cidade Gurupi. *diferente dos 7\% sugerido pela III Consenso de Pressão Arterial da Sociedade Brasileira de Cardiologia $(p<0,05)$. † diferente do grupo 15-16 anos $(p<0,05)$. Foi utilizado o teste de Poisson: "duas amostras", para comparação inter-grupo, e "uma amostra" para comparação com a referência do III Consenso de Pressão Arterial da Sociedade Brasileira de Cardiologia, para crianças e adolescentes.

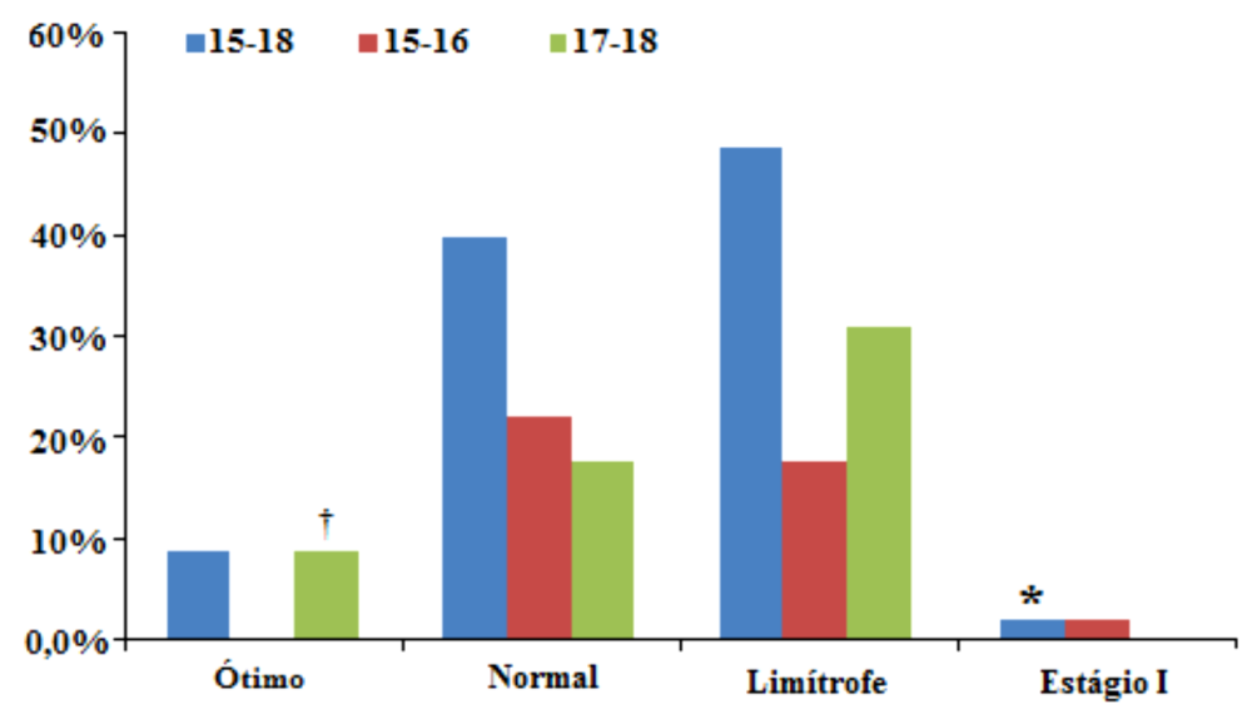

O percentual de sujeitos, de acordo com a classificação do índice de massa corporal, está ilustrado no gráfico 2. Os valores mostraram que a prevalência de obesidade foi de 2,2\% entre sujeitos de 15-18 anos, esse valor encontrado é significativamente abaixo do valor de $17 \%$ encontrado pela pesquisa Vigitel. ${ }^{6}$ Quando fragmentado esse grupo, notouse que o valor ficou centrado no grupo com idade de 15-16 anos.

Já a prevalência de sobrepeso, foi de $6,7 \%$ entre os sujeitos estudados. Esse percentual encontrado é significativamente abaixo do valor de $60 \%$ encontrados pela pesquisa Vigitel. ${ }^{6}$ Quando subdividido esse grupo, percebe-se que o percentual ficou 
centrado no grupo com idade 17-18 anos. Constatou-se que os valores encontrados de obesidade e sobrepeso, ainda são menores do que os indivíduos abaixo do peso (8,9\%). A partir disso, é notório que a obesidade concentrou-se nos sujeitos mais novos (15-16 anos) e o percentual de indivíduos identificados com sobrepeso e abaixo do peso, agrupou em sujeitos mais velhos (17-18 anos).

Gráfico 2 - Percentuais da classificação do índice de massa corporal de alunos do sexo masculino de unidade escolares de ensino médio público da cidade Gurupi ${ }^{\star}$ diferente do percentual encontrado (sobrepeso de $60 \%$ e obesidade de 17\%) na pesquisa do Ministério da Saúde Vigitel ${ }^{6}$ para faixa etária de 12 a 18 anos $(p<0,05)$. Foi utilizado o teste de Poisson: "duas amostras", para comparação intergrupo, e "uma amostra' para comparação com a referência do Ministério da Saúde, para faixa etária de 12 a 18 anos.

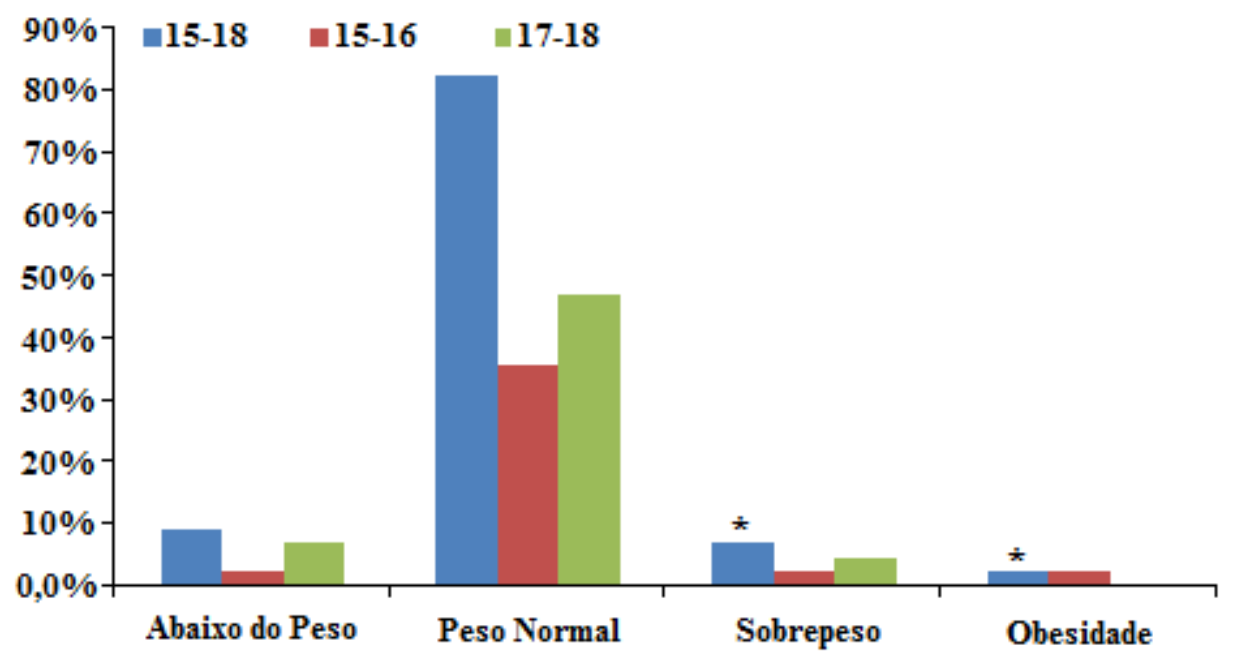

Quanto aos resultados do nível de atividade física esboçados no gráfico 3 , a prevalência do sedentarismo foi $0 \%$, esse valor encontrado é significativamente abaixo do valor de $15,7 \%$ encontrado pela pesquisa Vigitel. ${ }^{6}$ Foram encontrados $44,4 \%$ indivíduos entre 15-18 anos muito ativos, tendo uma porcentagem maior de $28,9 \%$ no grupo de $17-18$ anos. Já os indivíduos considerados ativos foi 35,6\%. Também foi encontrado indivíduos irregularmente ativos, sendo irregularmente ativo " $\mathrm{A}$ " $13,3 \%$ e o irregularmente ativo "B" $6,7 \%$.

Identificou-se que não houve diferença significativa entre os grupos de idade, no entanto os valores tiveram uma tendência maior no grupo de 17-18 anos. 
Gráfico 3 - Percentuais da classificação do nível de atividade física de alunos do sexo masculino de unidade escolares de ensino médio público da cidade Gurupi. ${ }^{*}$ diferente do percentual encontrado (inatividade física de $15,7 \%)$ na pesquisa do Ministério da Saúde Vigitel ${ }^{6}$ para faixa etária de 12 a 18 anos $(p<0,05)$.Foi utilizado o teste de Poisson: "duas amostra", para comparação inter-grupo, e "uma amostra' para comparação com a referência do Ministério da Saúde, para faixa etária de 12 a 18 anos.

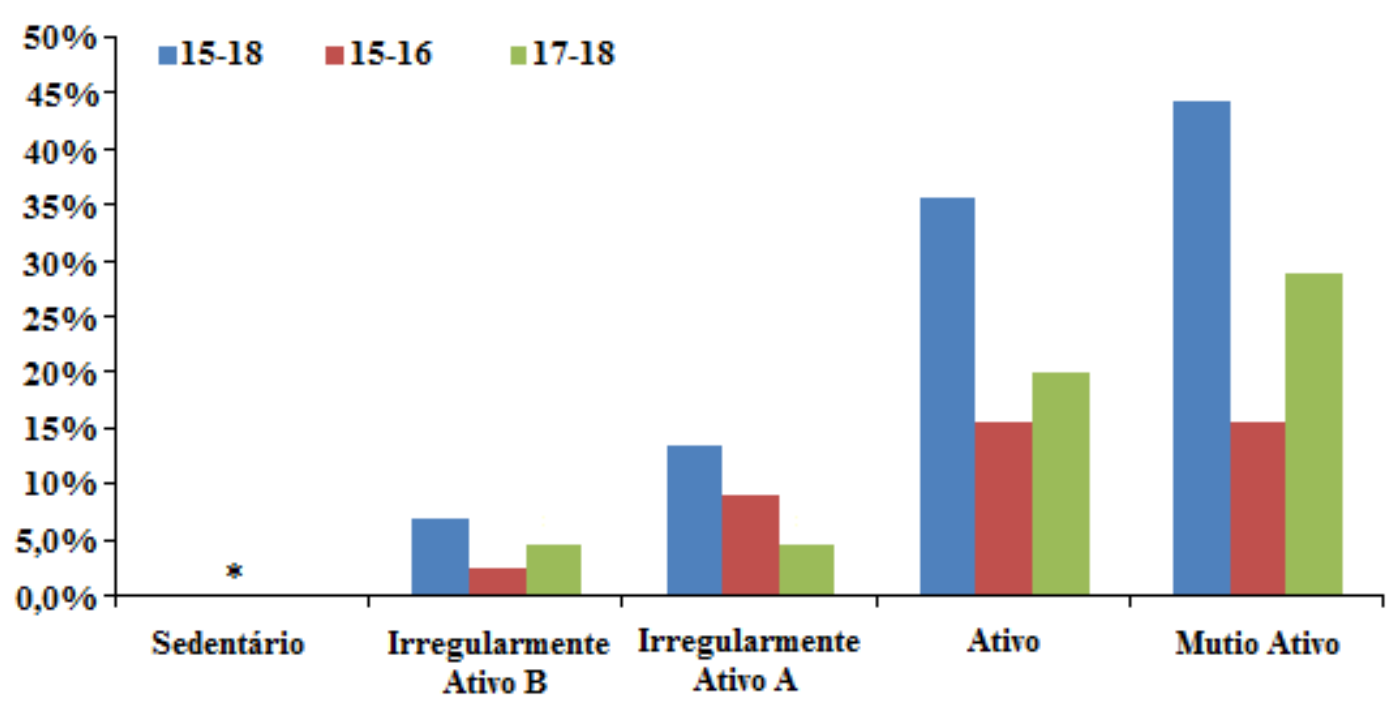

\section{DISCUSSÁO}

Nesta pesquisa a prevalência de obesidade entre adolescentes do sexo masculino foi $2,2 \%$. Os estudos que contribuíram como referências para esta pesquisa revelaram prevalências de obesidade em adolescentes que variaram de 2,6 e 13, 4\%. ${ }^{78}$ No entanto, no estudo de Abrantes ${ }^{7}$ que faz uma comparação entre região sudeste e nordeste, apresenta uma prevalência de obesidade de 1,7\% no nordeste e de $4,2 \%$ no sudeste, comprovando, que a prevalência de obesidade é significativamente menor na região nordeste. Nota-se assim, que o resultado encontrado na região nordeste, que se localiza mais próxima a região norte, possui uma semelhança com o resultado encontrado nesse estudo.

Em uma pesquisa feita em 15 capitais pelo Instituto Nacional do Câncer $^{9}$ a prevalência de obesidade no Rio de Janeiro foi 12,9\% e em Belém de 8,7\%. Revelando que o valor encontrado no presente estudo é baixo em relação aos valores encontrados no estudo citado acima.

Os indivíduos que eventualmente manter-se no estado de obesidade poderão acarretar alguns danos à saúde como surgimento precoce de hipertensão arterial, dislipidemias, diabetes tipo 2, distúrbios emocionais, alterações no aparelho locomotor. ${ }^{10}$ 
Concomitantemente, o único indivíduo considerado hipertenso foi também o único considerado obeso, corroborando com a literatura, onde a obesidade é um fator de risco para aquisição da hipertensão. ${ }^{11}$

A prevalência de hipertensão em adolescentes segundo Salgado ${ }^{12}$ varia de $1 \%$ a $13 \%$, ressaltando que em estudos que a pressão arterial é medida em apenas uma vez a taxa é de $1 \%$ e nos estudos em que a medida é realizada em várias vezes a taxa é $13 \%$. Em contra partida, o referido estudo mensurou a pressão arterial apenas uma vez e sua prevalência foi de $2,2 \%$ isso representa mais do que o dobro do valor encontrado na literatura.

O valor de $48,9 \%$ sujeitos considerados limítrofes (pré-hipertensão) foi o mesmo valor de sujeitos considerados normais. A pré-hipertensão é considera por Ortega ${ }^{13} \mathrm{como}$ precursora da hipertensão arterial e está relacionada à elevação do desenvolvimento de outras doenças cardiovasculares. Surgindo assim uma maior atenção com esse percentual, pois a hipertensão arterial é uma doença silenciosa, que não mostra sintomas evidentes para sua identificação.

Todavia neste estudo não foram identificados sujeitos sedentários, o que é favorável para a população. Isso pode ser decorrente do município de Gurupi ser pequeno em comparação às grandes metrópoles, em que a maioria dos alunos desloca-se de carros ou transporte público para escola.

Os resultados corroboram com a pesquisa feita pela Vigitel ${ }^{6}$ que revela uma diminuição da inatividade física, principalmente entre homens de 16\% em 2009 para 14,1\% em 2011. Apresenta também que a cidade de Palmas, capital do estado do Tocantins, fica $223 \mathrm{Km}$ de Gurupi, encontra-se entre as capitais que mais prática atividade física com um percentual de $34 \%$, comparado com Porto Velho, capital que menos pratica atividade física com percentual de $26 \%$.

Estudo feito por Gomes $^{14}$ na região sul do Rio de Janeiro apresenta que a prevalência de sedentarismo em adolescentes e adultos do sexo masculino, foi 59,8\%. Já no estudo feito em Gurupi por Rodrigues ${ }^{15}$ a prevalência de sedentarismo foi $29,9 \%$ em adultos do sexo masculino, resultados semelhantes com a pesquisa do Instituto Nacional do Câncer ${ }^{9}$ que encontrou a menor prevalência de sedentários em Belém $(28,2 \%)$, estado que está situado também na região norte do País.

De acordo com Malta ${ }^{16}$ indivíduos que têm o costume de realizar atividades físicas amenizam os ricos de doenças que o sedentarismo proporciona como doenças 
cardiovasculares, diabetes II, cânceres de mama, colo e reto. A partir disso, os indivíduos que se mantiverem ativos não irão sofrer futuramente os prejuízos de um sujeito sedentário. Mediante ao exposto, nota-se que foram encontrados valores positivos e significativos nos jovens com faixa etária de 15-16 anos e 17-18 anos das Escolas e Centros de Ensino Médio Regular da educação pública de Gurupi-TO, ao comparar com as pesquisas apresentadas. No entanto, para essas taxas permanecerem favoráveis à população é necessário conscientizar os alunos a adquirir hábitos saudáveis. De acordo com Guedes ${ }^{17}$ hábitos da prática de atividade física, incluídos na infância e na adolescência, provavelmente manter-se-á na idade adulta.

O ambiente escolar é um local determinante para preparar adolescentes para o futuro, e nas aulas de Educação Física surge ocasiões apropriadas para o professor, conscientizar os alunos a adquirir hábitos saudáveis para melhoria da qualidade de vida.

\section{CONCLUSÁO}

Conclui-se que a prevalência de obesidade, hipertensão e sedentarismo foram abaixo do encontrado em relação a outros estudos pelo país. Confirmando assim a hipótese levantada no início deste estudo, com exceção dos valores de indivíduos classificados como limítrofes. Sobre essas informações entende-se que é necessária uma atenção maior à quantidade de sujeitos considerados com a pressão arterial como limítrofes, pois este é um fator para aquisição da hipertensão arterial.

Outro dado importante é a quantidade de sujeitos abaixo do peso que se deve a essa faixa etária de 15 a 19 anos, ser mais preocupada com questões de estética. Esse dado não pode ser esquecido, pois sua manutenção poderá desenvolver o aparecimento de problemas de saúde como desnutrição, anorexia, anemia entre outros.

Com o findar deste estudo, sugere-se a necessidade de novas pesquisas com uma amostra maior, com pelo menos três visitas para mensuração da pressão arterial e que leve em considerações os aspectos socioeconômicos e hábitos alimentares para melhor interpretação dos resultados encontrados.

\section{REFERÉNCIAS}

1. Rique ABR, Soares EA e Meirelles CM. Nutrição e exercício na prevenção e controle das doenças cardiovasculares. Revista Brasileira Medicina Esporte. Vol. 8, № 6 - Nov/Dez, 2002. 
DOI: 10.18606/2318-1419/amazonia.sci.health.v7n3p12-21 Revista Amazônia: Science \& Health

2019, Vol. 7, № 3 .

ISSN: 2318-1419
Rodrigues JBA. Gross DGL.

Prevalência de obesidade, hipertensão e sedentarismo em alunos do ensino médio da rede pública da cidade de Gurupi.

2. Oehlschlaeger MHK, Pinheiro RT, Horta B, Gelatti C e San'Tana P. Prevalência e fatores associados ao sedentarismo em adolescentes de área urbana. Revista Saúde Publica. p.159, 2003

3. Fisberg, M. Atualização em obesidade na infância e na adolescência. São Paulo: Editora Atheneu, 2004, $218 p$.

4. Ministério da saúde. O Que é a hipertensão arterial? [Acesso em 10 de setembro de 2012] Disponível em: http://portal.saude.gov.br/portal/saude/visualizar texto.cfm?idtxt=36873\&janela=1

5. Barreto ACLG, Ribeiro LG. Determinação do tamanho amostral. Fitness e Performance Journal [S.I.], v. 3, n. 3, p. 124. 2004.

6. Vigitel. Secretaria de Vigilância em Saúde, Secretaria de Gestão Estratégica e Participativa. - Brasília: Ministério da Saúde, 2011.

7. Abrantes, MM.; Lamounier, JA, Colosimo, EA. Prevalência de sobrepeso e obesidade em crianças e adolescentes das regiões Sudeste e Nordeste. Jornal de Pediatria - Vol. 78, №4, 2002

8. Andrade, DE. Prevalência de Sobrepeso e Obesidade em Crianças de Escolas Publicas e Privadas do Ensino Fundamental da Cidade de Franca - SP e Alguns Fatores de Risco Associados. $2006.73 f$. Dissertação (Mestrado) - Faculdade de Medicina de Ribeirão Preto, Universidade de São Paulo, Ribeirão Preto 2007.

9. Instituto nacional do câncer (INCA). Inquérito Domiciliar sobre Comportamentos de Risco e Morbidade Referida de Doenças e não transmissíveis: Brasil, 15 capitais e Distrito Federal, 2002-2003. Rio de Janeiro: 2004.

10. Enes, CC. Obesidade na adolescência e seus principais fatores determinantes. Rev Bras Epidemiol 2010; 13(1): 163-71

11. Simonetti JP, Batista L, Carvalho, LR Hábitos de saúde e fatores de risco em pacientes hipertensos. Rev. Latino americana. Enfermagem 2002 maio-junho; 10(3):415-22

12. Salgado CM, Carvalhaes, JTA Hipertensão arterial na infância. Jornal de Pediatria - Vol.79, Supl.1, 2003

13. Ortega KC, Ginani GF, Silva GV, Mion Jr. Pré-hipertensão: conceito, epidemiologia e o que falam as diretrizes D 84 Rev Bras Hipertens vol.16(2):83-86, 2009.

14. Gomes V B, Siqueira KS, Sichieri R. Atividade física em uma amostra probabilística da população do Município do Rio de Janeiro. Cad Saúde Pública 2001;17:969-76

15. Rodrigues E S R, Cheik NC, Mayeret A F. Atividade física e tabagismo em universitários .Rev Saúde Pública 2008;42(4):672-8

16. Malta, DC. Padrão de atividade física em adultos brasileiros: resultados de um inquérito por entrevistas telefônicas, 2006. Epidemiol. Serv. Saúde, Brasília, 18(1):7-16, jan-mar 2009

17. Guedes DP, Guedes ERP, Barbosa, DS, Oliveira J A, Stanganellli L C R. Fatores de Risco Cardiovasculares em Adolescentes: Indicadores Biológicos e Comportamentais. Arquivos Brasileiros de Cardiologia - Volume 86, № 6, Junho 2006 